\title{
The walls of the spores and basidia of Tricholoma found to be cyanophilic
}

\author{
Harri Harmaja \\ Botanical Museum, University of Helsinki, SF-00170 Helsinki, Finland

\begin{abstract}
HARMAJA, H. 1976: The walls of the spores and basidia of Tricholoma found to be cyanophilic. - Karstenia 15:23-24.

The author observed that the walls of the spores and basidia of all of those nine species (including the type) of the genus Tricholoma (Fr.) Staude (Agaricales) examined by him are weakly cyanophilic. In the literature these structures have been considered cyanophobic.
\end{abstract}

SINGER (1972) and I myself (HARMAJA 1974) have stated that the spore wall is cyanophobic, or acyanophilous, in those species of the genus Tricholoma (Fr.) Staude (Agaricales) which were examined for the response of that structure to cotton blue (the wall of the exceptional "sclerospores" of some species was, however, found cyanophilic and dextrinoid; see HARMAJA 1974). KOTLABA \& POUZAR (1964) reported that in their preliminary survey they did not find cyanophilic spore walls in the family Tricholomataceae Roze.

Recently I re-examined the hymenial elements of Tricholoma in cotton blue, and observed that in fact the spore wall in all species studied is weakly (or very weakly) but without any doubt cyanophilic. The degree of cyanophily varies slightly with species, being most distinct in $T$. bufonium and $T$. imbricatum of the species examined. Anyway, the degree of cotton blue absorbing in Tricholoma appears somewhat lower than e.g. in the pinkspored species of Clitocybe recently transferred to Lepista (HARMAJA 1976). It is probable that in Tricholoma the wall of the hilar appendage, too, is cyanophilic which would be exceptional among pale-spored agarics with cyanophilic spore walls. In all species examined in the present study, also the basidial wall, including that of the sterigmata, proved weakly cyanophilic. This is apparently the first report of cyanophilic basidia in the order Agaricales.
The previous observations with results which are the contrary to the present ones, are thus considered erroneous, most probably due to examination not intensive enough. Always when the spore wall at first appears cyanophobic, it is of utmost importance to carefully check under high magnification the response of the wall by examining occasional empty (often collapsed) spores in which the cyanophily of the contents does not interfere with the observing of the character of the wall!

The nine species of Tricholoma examined by me include those four reported in SINGER 1972 and are: T. albobrunneum (Fr.) Kumm., T. album (Fr.) Quél., T. aurantium (Fr.) Ricken, T. bufonium (Fr.) Gillet, T. caligatum (Viv.) Ricken, T. flavobrunneum (Fr.) Kumm., T. flavovirens ( $\mathrm{Fr}$.) Lund. (the type species of the genus!), T. imbricatum (Fr.) Kumm., and T. saponaceum (Fr.) Kumm.

These new observations are no doubt of importance for the taxonomy of the family Tricholomataceae. It appears, for instance, that (1) the genera Clitocybe (Fr.) Staude sensu HARMAJA 1976 and Tricholoma are separated by a hiatus more distinct than supposed before, and (2) the genera Lepista (Fr.) W. G. Smith and Tricholoma are, to the contrary, related more closely than believed in recent times. In the light of these results, the closest relatives of Clitocybe (sensu strictissimo) with its cyanophobic spore wall should perhaps be sought among Omphalina 
Quél., Pleurotus (Fr.) Kumm. and allied genera, and Hygrophoraceae Roze.

Even if the recent use of the cotton blue staining method has provided new and apparently more natural views about the generic limits and clarified relationships between genera in the family Tricholomataceae, all problems have by no means been solved yet and the taxonomy of the family still needs much research work.

\section{REFERENCES}

HARMAJA, H. 1974: A revision of the generic limit between Clitocybe and Lepista. - Karstenia 14: 82-92.

- 1976: A further revision of the generic limit between Lepista and Clitocybe. - Karstenia 15:13-15.
KOTLABA, F. \& POUZAR, Z. 1964: Preliminary results on the staining of spores and other structures of Homobasidiomycetes in cotton blue and its importance for taxonomy. Feddes Repert. 69: 131-142.

SINGER, R. 1972: Cyanophilous spore walls in the Agaricales and agaricoid Basidiomycetes. Mycologia 64: 822-829. 